\title{
Variáveis fisiológicas em cães submetidos à infusão contínua de diferentes doses de propofol
}

\author{
Physiologic parameters in dogs anesthetized with different rates \\ of continuous infusion of propofol
}

\section{Patrícia Cristina Ferro $^{1}$ Newton Nunes ${ }^{2}$ Danielli Parrilha de Paula ${ }^{1}$ Celina Tie Nishimori ${ }^{1}$ Elaine Dione Venêga da Conceição ${ }^{1}$ Piedad Natália Henao Guerrero ${ }^{3}$ Lívia Moraes Arruda ${ }^{1}$}

RESUMO

A fim de determinar possíveis alterações nos principais parâmetros fisiológicos determinados pela infusão contínua de propofol, em diferentes doses, foram utilizados 24 cães adultos distribuídos aleatoriamente em 3 grupos (P2, P4 e P8). Os animais foram induzidos à anestesia pela administração intravenosa de propofol $(10 \mathrm{mg} / \mathrm{kg})$ e, ato contínuo, os cães receberam o anestésico, em infusão contínua nas doses de $0,2 \mathrm{mg} /$ $\mathrm{kg} / \mathrm{min}$ (P2), 0,4mg/kg/min (P4) e 0,8mg/kg/min (P8). As mensurações dos valores das variáveis cardiorrespiratórias [freqüência cardíaca (FC); pressões arteriais sistólica, diastólica e média (PAS, PAD e PAM, respectivamente); eletrocardiografia e freqüência respiratória (f)] e temperatura retal (T) foram realizadas antes da aplicação do fármaco (M0) e após 10, 20, 30, 40 e 50 minutos do início da infusão contínua. Os dados numéricos das variáveis estudadas foram submetidos à Análise de Variância (ANOVA) seguida pelo Teste $F(P<0,05)$. Para $F C$, observaramse diferenças entre os grupos no M20 (P2: $91 \pm$ 14,92; P4: 113 \pm 17,18; P8: $120 \pm$ 14,84), M30 (P2: $89 \pm$ 13,79; P4: $110 \pm$ 14,3; P8: $114 \pm$ 10,89) e em M40 (P2: $88 \pm$ 17,3; P4: $103 \pm$ 16; P8: $109 \pm 8,2)$, que podem ser justificadas por P2 apresentar menor redução da PAM, não havendo a necessidade de aumento da FC para estabilizar o débito cardíaco $e$ consequentemente para manter a PA. A PAS registrou redução de $26,9 \%$ para $P 2,23,6 \%$ para $P 4$ e $30,6 \%$ para $P 8$. Para $P A D$ foram observados decréscimos de $36,2 \%$ para $P 2 ; 38,1$ para $P 4$ e $52,7 \%$ para $P 8$, enquanto para PAM a diminuição foi de $32 \%$ para $P 2$, 26,3\% para $P 4$ e $38,4 \%$ para $P 8$. Essas reduções poderiam ser ocasionadas pela diminuição da resistência periférica vascular. Concluiu-se que as reduções ocorridas na PAS, PAD, PAM e f são dependentes da dose de infusão do fármaco, que também proporcionou discreta redução na temperatura retal nos três grupos.

Palavras-chave: propofol, anestesia intravenosa, cães, monitoração.

\begin{abstract}
The aim of this article was to establish the correlation between different rates of continuous infusion of propofol and the alterations that might occur with the physiologic parameters most commonly measured by the anesthesiologists. Twenty four adult dogs were randomly divided into 3 groups (P2, P4, P8). All the animals were induced with propofol $(10 \mathrm{mg} / \mathrm{kg})$, followed immediately by the continuous infusion of the agent: $0.2 \mathrm{mg} / \mathrm{kg} / \mathrm{min}(P 2)$, $0.4 \mathrm{mg} / \mathrm{kg} / \mathrm{min}(\mathrm{P} 4)$ and $0.8 \mathrm{mg} / \mathrm{kg} / \mathrm{min}(P 8)$. The heart rate (HR), systolic, diastolic and mean arterial pressures (SAP, DAP and MAP), electrocardiography (ECG), respiratory rate $(R R)$, and body temperature $(T)$ were measured before any drug administration (MO) and 10, 20, 30, 40 and 50 minutes after the start of the continuous infusion of propofol. The numerical data were submitted to Profile of Variance followed by $F$ Test $(P<0.05)$. The HR showed differences among groups at M20 (P2: $91 \pm$ 14,92; P4: $113 \pm$ 17,18; P8: $120 \pm$ 14,84), M30 (P2: 89 \pm 13,79; P4: $110 \pm 14,3 ; P 8: 114 \pm 10,89)$, and M40 (P2: $88 \pm 17,3$; P4: $103 \pm 16$; P8: $109 \pm 8,2$ ), wich could be justified because $P 2$ demonstrated minor reduction of MAP. There is no need of HR increasing to establish the cardiac output, and consequently, to maintain the AP. A reduction in SAP about $26,9 \%$ to $P 2,23,6 \%$ to $P 4$ and $30,6 \%$ to $P 8$, in PAP about $36,2 \%$ to $P 2 ; 38,1$ to $P 4$ and $52,7 \%$ to P8I; and in MAP about $32 \%$ to P2, $26,3 \%$ to $P 4$ and $38,4 \%$ to $P 8$ were observed. These reductions could be related to the decrease of peripheral vascular resistance. It was possible to conclude that the decrease observed in SAP, DAP, MAP and RR are dependent on the infusion rate of propofol, which also caused reduction of the rectal temperature in the 3 groups.
\end{abstract}

Key words: propofol, intravenous anesthesia, dogs, monitoring.

${ }^{1}$ Faculdade de Ciências Agrárias e Veterinárias (UNESP) Campus Jaboticabal, Jaboticabal, SP, Brasil.

${ }^{2}$ Departamento de Clínica e Cirurgia Veterinária, Faculdade de Ciências Agrárias e Veterinárias, UNESP, Campus Jaboticabal, Via de Acesso Prof. Paulo Donato Castellane s/n, Zona Rural, 14.884-900, Jaboticabal, SP, Brasil. E-mail: newton@fcav.unesp.br. Autor para correspondência.

${ }^{3}$ Departamento de Ciências Clínicas, Faculdade de Medicina Veterinária, Universidade do Estado de Kansas, Manhattan, KS, EUA. 


\section{INTRODUÇÃO}

A infusão contínua de fármacos como o propofol apresenta-se como uma alternativa importante em substituição à anestesia inalatória, por apresentar boa estabilidade hemodinâmica, indução e recuperação anestésica rápidas, poucos efeitos adversos e ausência de efeito cumulativo. Dessa forma, trabalhos realizados com este fármaco, proporcionam embasamento prático para que seja cada vez mais freqüente seu emprego na rotina anestésica.

Com o propofol, a indução e recuperação da anestesia geralmente ocorrem de forma satisfatória sendo possível verificar ausência de fenômenos excitatórios, quando sedativos são utilizados na medicação pré-anestésica. MORGAN \& LEGGE (1989) observaram, ocasionalmente, excitação durante a indução com propofol, em cães e gatos sem prémedicação.

Nos procedimentos anestésicos com infusão contínua deste fármaco são indicadas taxas de 0,15 a $0,4 \mathrm{mg} / \mathrm{kg} / \mathrm{min}$, enquanto a infusão com bolus intermitente requer administração de 0,5 a $2 \mathrm{mg} / \mathrm{kg}$ para cães (THURMON et al., 1996).

PIRES et al. (2000) utilizaram para infusão contínua, em cães, doses de 0,3 a $0,8 \mathrm{mg} / \mathrm{kg} / \mathrm{min}$, conforme a medicação pré-anestésica e a associação com sedativos e analgésicos. HALL \& CHAMBERS (1987) empregaram propofol continuamente e demonstraram que a infusão na taxa de $0,4 \mathrm{mg} / \mathrm{kg} / \mathrm{min}$ produz anestesia satisfatória para procedimentos cirúrgicos em cães pré-tratados com acepromazina.

No sistema respiratório, o propofol causa depressão podendo ocorrer apnéia transitória após sua administração, diminuição do volume minuto (VM) e da freqüência respiratória $(f)$, com aumento da pressão parcial arterial de dióxido de carbono $\left(\mathrm{PaCO}_{2}\right)$ e diminuição da pressão parcial arterial de oxigênio $\left(\mathrm{PaO}_{2}\right)$, sendo a incidência destes efeitos diretamente proporcional à dose administrada (FANTONI et al.,1996).

Já no sistema cardiovascular, o propofol parece não afetar a sensibilidade dos barorreceptores. Provoca redução nas pressões arteriais sistólica, diastólica e média (ANTUNES, 1999), ocasionando hipotensão sistêmica resultante da redução da resistência vascular periférica. A redução na pressão arterial é proporcional ao aumento da concentração plasmática do agente anestésico (WHITWAM et al., 2000).

Essa ação depressora do fármaco está relacionada a seus efeitos diretos sobre o miocárdio e a vasodilatação arterial e venosa (FANTONI, 2002a). KEEGAN \& GREENE (1993) relataram redução da FC durante anestesia com propofol, caracterizando o efeito cronotrópico negativo (QUANDT et al., 1998). No entanto, AGUIAR et al. (1993) observaram redução deste parâmetro, especialmente após 20 minutos de anestesia, coincidindo com a diminuição dos valores de pressão arterial. Segundo FANTONI (2002a), tanto taquicardia quanto bradicardia podem ser verificados com esse agente.

GOODCHILD \& SERRAO (1989) concluíram que a anestesia com propofol pode ser acompanhada pela diminuição do débito cardíaco, secundariamente a redução da pré-carga causada pelo efeito direto de vasodilatação, sendo que o débito cardíaco e a pressão arterial podem ser preservados, desde que a pré-carga seja mantida.

O propofol produz depressão do sistema nervoso central (SNC) de maneira dose-dependente (SHORT; BUFALARI, 1999). Esse quadro é induzido através do potente efeito agonista sobre a transmissão GABA-érgica, mediado pela ligação ao receptor $\mathrm{GABA}_{\mathrm{A}}$ e por reduzir a atividade metabólica cerebral (THURMON et al., 1996).

Sendo assim, objetivou-se, com este trabalho, avaliar possíveis alterações em parâmetros fisiológicos durante a administração em infusão contínua de diferentes doses de propofol, em cães, de modo a propiciar informações complementares, relativas à anestesia intravenosa aos profissionais que militam e na prática clínico-cirúrgica, em especial aos anestesiologistas veterinários.

\section{MATERIAL E MÉTODOS}

Este experimento foi aprovado pela Comissão de Ética Bem Estar Animal da FCAV/Unesp e, terminado o estudo, os animais foram oferecidos para adoção.

Foram utilizados 24 cães adultos, 12 machos e 12 fêmeas, sem raça definida, evitando-se fêmeas prenhes ou em estro, considerados hígidos após exames clínicos e laboratoriais (hemograma completo, urinálise, perfis bioquímicos renal e hepático), com peso variando entre 6 e $20 \mathrm{~kg}$.

Os animais foram mantidos em canis individuais do Hospital Veterinário "Governador Laudo Natel”, da Faculdade de Ciências Agrárias e Veterinárias - FCAV/UNESP, Câmpus Jaboticabal, SP, sendo fornecida ração comercial e água "ad libitum”.

Após serem selecionados aleatoriamente, os cães foram distribuídos em 3 grupos de 8 animais, denominados P2, P4 e P8.

Para todos os animais, induziu-se a anestesia geral pela administração intravenosa de 
propofola $^{\text {a }}$ na dose de $10 \mathrm{mg} / \mathrm{kg}$. Em seguida, iniciou-se a infusão contínua do fármaco por meio de bomba de infusão ${ }^{\mathrm{b}}$, empregando-se as doses de $0,2 \mathrm{mg} / \mathrm{kg} / \mathrm{min}$ para o grupo P2; 0,4mg/kg/min para o P4 e 0,8 mg/kg/ min para o P8. O fármaco foi administrado durante 50 minutos.

Os valores de freqüência cardíaca (FC) e o traçado eletrocardiográfico (em derivação DII) foram obtidos por meio de monitor multiparamétrico ${ }^{c}$. As pressões arteriais sistólica, diastólica e média (PAS, PAD e PAM, respectivamente) foram obtidas empregando-se o mesmo equipamento, cujo manguito foi adaptado ao membro torácico direito, acima da articulação do cotovelo, para mensuração pelo método oscilométrico. O mesmo monitor também foi utilizado para a mensuração da temperatura retal $(\mathrm{T})$. Neste caso, o sensor foi introduzido no reto dos animais.

A freqüência respiratória $(f)$ foi obtida pela observação dos movimentos do gradil costal. A observação foi efetuada durante 60 segundos obtendose dessa forma o número de movimentos por minuto (mov/min).

Também foram realizadas observações, com o objetivo de diagnosticar a presença de efeitos neuromusculares, como opistótomo, movimentos de pedalagem e tremores musculares, conforme relatado por QUANDT et al (1998).

A primeira observação foi realizada imediatamente antes da indução com propofol (M0). As demais mensurações foram efetuadas 10 (M10), 20 (M20), 30 (M30), 40 (M40) e 50 (M50) minutos após a indução anestésica. A avaliação estatística foi efetuada por meio de Análise de Variância (ANOVA), seguida pelo teste de Tukey $(\mathrm{p}<0,05)$.

\section{RESULTADOS E DISCUSSÃO}

Em relação à freqüência cardíaca, foi possível observar redução das médias dessa variável nos três grupos, sendo que o aumento das médias da FC no grupo P8, após a indução pode ser justificado pela possível estimulação simpática durante a infusão de propofol (FANTONI, 2002a; HALL; CHAMBERS, 1987).

Apesar dessas alterações, pode-se afirmar que a FC manteve-se dentro do intervalo considerado fisiológico para a espécie (TILLEY, 1992). Essa estabilidade também foi descrita por QUANDT et al. (1998), em cães, após trinta minutos de anestesia com esse fármaco. Já ROBERTS et al. (1988) relataram redução lenta dos valores de FC, durante os cinco primeiros minutos de infusão com propofol, com posterior estabilização nos momentos seguintes. No entanto, essas observações não foram verificadas por ANTUNES (1999) e DUKE (1995) que afirmaram ter o propofol uma ação cronotrópica negativa, oriunda de um efeito vagotônico central ou ação simpatolítica. Desta forma, acredita-se que a ausência de alterações na FC, após a indução com propofol, tenha sido decorrente da taxa de administração.

As diferenças observadas entre as médias dos grupos nos momentos M20, M30 e M40, para esta variável podem ser justificadas pelo fato do P2 apresentar redução menor dos valores médios de pressão arterial média, não sendo necessário o aumento da freqüência cardíaca na tentativa de estabilizar o débito cardíaco e, conseqüentemente, manter a pressão arterial. Observações semelhantes foram relatadas por COATES et al. (1997), os quais afirmaram que a administração de propofol não acarreta alteração da sensibilidade dos barorreceptores e nem o número de receptores muscarínicos. Portanto, o aumento da freqüência cardíaca nos pacientes submetidos a infusão de propofol pode ser conseqüência da redução da pressão arterial, causada pelo fármaco.

Na eletrocardiografia não foram detectadas alterações durante os procedimentos, nos três grupos, o que está de acordo com WATKINS et al. (1987), que descreveram que o desenvolvimento de arritmias cardíacas após a administração de propofol são raras.

Para as PAS, vinte minutos após a indução anestésica e início da infusão contínua de propofol observou-se a redução desse parâmetro em torno de 26,9\% para P2; 23,6\% para $\mathrm{P} 4$ e 30,6\% para P8. Para a PAD ocorreu um decréscimo maior sendo de $36,2 \%$; $38,1 \%$ e $52,7 \%$ para os mesmos grupos, respectivamente. As porcentagens de redução na PAM observadas foram 32\%; $26,3 \%$ e $38,4 \%$ para as doses de infusão de 0,$2 ; 0,4$ e $0,8 \mathrm{mg} / \mathrm{kg} / \mathrm{min}$, respectivamente. Este achado foi relatado por BUFALARI et al. (1996), que reportaram redução de, aproximadamente, 26\% na PAD, 20 minutos após a administração de $6,6 \mathrm{mg} / \mathrm{kg}$ de propofol em cães, sendo esta redução relacionada à diminuição da resistência vascular periférica, efeito este conhecido do propofol (MUIR III; GADAWSKI, 1998; QUANDT et al., 1998). GUERRERO (2003) também observou em cães prétratados com levomepromazina, que após a indução com propofol, houve redução nos valores de pressão arterial, entretanto, deve-se ressaltar o efeito hipotensivo da fenotiazina.

É interessante salientar que os menores valores de pressão arterial foram encontrados no P8 e os mais altos no P2 (Tabela 1), o que pode ser justificado devido à redução da pressão ser dependente da dose (KARSKI et al., 1994). 
HALL \& CHAMBERS (1987) trabalhando com cães pré-medicados com acepromazina e submetidos à infusão contínua de propofol a uma taxa de $0,5 \mathrm{mg} / \mathrm{kg} / \mathrm{min}$ observaram hipotensão severa em 3 animais, levando à interrupção do procedimento. No entanto, mesmo administrando $0,8 \mathrm{mg} / \mathrm{kg} / \mathrm{min}$ de propofol neste estudo, dose superior à testada pelos autores citados, não se observou quadro hipotensivo semelhante ao relatado, o que pode ser explicado devido ao fato de os animais utilizados não terem sido pré-medicados.

Para a $f$, não houve diferença entre os grupos, nem entre os momentos dentro de cada grupo. A explicação pode residir na grande variabilidade deste parâmetro, atestada pelos elevados coeficientes de variação encontrados. Ao se observar a tabela 1, é possível perceber redução na $f$ nos três grupos após a indução e início da infusão, porém com maior efetividade para os grupos tratados com as maiores doses. A redução da variável nos três grupos foi de $30,9 \%, 72,5 \%$ e $70,6 \%$ para P2, P4 e P8, respectivamente, comprovando seu efeito depressor sobre a função respiratória (FANTONI et al.,1996).

Relatos de apnéia são descritos durante a indução com propofol, sendo necessário, em alguns casos, assistência à respiração durante alguns momentos (HALL; CHAMBERS, 1987; MORGAN; LEGGE, 1989; MUIR III; GADAWSKI, 1998; QUANDT et al., 1998). PIRES et al. (2000) observaram apnéia em apenas um animal na sua experimentação, enquanto no presente trabalho não houve casos de apnéia durante a indução com 10mg/ $\mathrm{kg}$ de propofol. Esse fato pode ser explicado pela velocidade de administração do fármaco, que, no trabalho realizado por PIRES et al. (2000), foi mais lenta do que a utilizada por MUIR III \& GADAWSKI (1998) indicando que a velocidade de administração do propofol na indução pode estar relacionada com depressão nos centros respiratórios, complementando a informação anterior de WATKINS et al. (1987), que acreditavam que a incidência de apnéia, na indução da anestesia, era maior com a administração rápida da dose.

Em relação à variável temperatura retal, os valores observados mantiveram-se dentro da faixa considerada fisiológica para espécie (FANTONI, 2002b) (Tabela 1), durante os 50 minutos de procedimento. No entanto, houve redução na temperatura retal dos cães por volta de $1,4^{\circ} \mathrm{C}$, que pode ser justificada pela diminuição da taxa de metabolismo basal, pela vasodilatação arterial e venosa causada pelo fármaco (FANTONI, 2002a).

Tabela 1 - Valores médios e desvios padrão $(\mathrm{x} \pm \mathrm{s}$ ) de FC (batimentos/minuto), PAS (mmHg), PAD (mmHg), PAM (mmHg), $f$ (movimentos/min) e T $\left({ }^{\circ} \mathrm{C}\right)$, em cães submetidos a diferentes doses de propofol $(0,2 ; 0,4$ ou $0,8 \mathrm{mg} / \mathrm{kg} / \mathrm{min}$, grupos P2, P4 e P8 respectivamente).

\begin{tabular}{|c|c|c|c|c|c|c|c|}
\hline \multirow{2}{*}{ Variáveis } & \multirow{2}{*}{ Grupos } & \multicolumn{6}{|c|}{ Momentos } \\
\hline & & M0 & M10 & M20 & M30 & M40 & M50 \\
\hline \multirow{3}{*}{ FC } & P2 & $109+16,74$ & $108+18,63$ & $91 \pm 14,92^{\mathrm{A}}$ & $89 \pm 13,79^{\mathrm{A}}$ & $88 \pm 17,27^{\mathrm{A}}$ & $86 \pm 17,22$ \\
\hline & P4 & $116 \pm 15,63$ & $115 \pm 15,24$ & $113 \pm 17,18^{\mathrm{B}}$ & $110 \pm 14,31^{\mathrm{B}}$ & $103 \pm 16,10^{\mathrm{B}}$ & $101 \pm 19,79$ \\
\hline & P8 & $107 \pm 22,17$ & $127 \pm 13,30$ & $120 \pm 14,84^{\mathrm{B}}$ & $114 \pm 10,91^{\text {В }}$ & $109 \pm 8,24^{\mathrm{B}}$ & $103 \pm 6,90$ \\
\hline \multirow{3}{*}{ PAS } & P2 & $130 \pm 13,99$ & $100 \pm 10,67$ & $95 \pm 6,94^{\mathrm{A}}$ & $94+13,43$ & $98 \pm \overline{7}, 98$ & $99+12,18$ \\
\hline & P4 & $140+22,78$ & $101+22,32$ & $107 \pm 8,86^{\mathrm{B}}$ & $102+12,27$ & $101 \pm 13,77$ & $106 \pm 16,32$ \\
\hline & P8 & $134 \pm 25,20$ & $96 \pm 5,12$ & $93 \pm 8,72^{\mathrm{A}}$ & $97 \pm 9,35$ & $98 \pm 10,60$ & $100 \pm 14,61$ \\
\hline \multirow{3}{*}{ PAD } & P2 & $80 \pm 8,62$ & $52 \pm 12,29^{\mathrm{A}}$ & $51 \pm 7,79^{\mathrm{A}}$ & $49 \pm 9,42$ & $50 \pm 8,57$ & $57 \pm 11,08$ \\
\hline & P4 & $76 \pm 19,22$ & $46 \pm 9,50$ & $47 \pm 9,70^{\mathrm{A}}$ & $50 \pm 19,93$ & $47 \pm 14,85$ & $55 \pm 15,95$ \\
\hline & P8 & $74 \pm 19,02$ & $36 \pm 4,50^{\text {В }}$ & $35 \pm 5,68^{\text {В }}$ & $36 \pm 8,67$ & $38+9,73$ & $42+9,82$ \\
\hline \multirow{3}{*}{ PAM } & P2 & $100+9,81$ & $70 \pm 11,22$ & $68+7,61$ & $67 \pm 12,45$ & $71 \pm 7,65$ & $72 \pm 10,48$ \\
\hline & P4 & $99+21,01$ & $69 \pm 14,84$ & $73 \pm 7,28^{\mathrm{A}}$ & $64+10,65$ & $70 \pm 15,68$ & $76 \pm 17,82$ \\
\hline & P8 & $99 \pm 21,37$ & $61 \pm 4,50$ & $61 \pm 6,58^{\mathrm{B}}$ & $61 \pm 8,72$ & $62 \pm 12,02$ & $65 \pm 10,37$ \\
\hline \multirow{3}{*}{$f$} & P2 & $42+38,18$ & $29+41,98$ & $28 \pm 36,21$ & $30 \pm 40,84$ & $23 \pm 19,87$ & $21 \pm 13,34$ \\
\hline & P4 & $40+43,24$ & $11 \pm 2,88$ & $12+3,85$ & $11+2,67$ & $12+3,67$ & $11 \pm 1,60$ \\
\hline & P8 & $51 \pm 50,41$ & $15+10,36$ & $11+9,07$ & $12 \pm 7,73$ & $13 \pm+9,90$ & $15+16,56$ \\
\hline \multirow{3}{*}{$\mathrm{T}$} & P2 & $38,9 \pm 0,40$ & $38,4 \pm 0,63$ & $38,1 \pm 0,59$ & $37,9 \pm 0,69$ & $37,6 \pm 0,74$ & $37,5 \pm 0,63$ \\
\hline & P4 & $38,8 \pm 0,40$ & $38,3 \pm 0,24$ & $38,2 \pm 0,26$ & $37,9 \pm 0,32$ & $37,8 \pm 0,41$ & $37,5 \pm 0,50$ \\
\hline & P8 & $38,9 \pm 0,75$ & $38,2 \pm 0,61$ & $38,2 \pm 0,54$ & $37,9 \pm 0,61$ & $37,7 \pm 0,67$ & $37,8 \pm 0,60$ \\
\hline
\end{tabular}

OBS: Não há diferença entre os momentos dentro dos grupos.

Médias seguidas por letras diferentes, nas colunas, diferem entre si pela Análise de Variância $(\mathrm{p}<0,05)$.

Ciência Rural, v.35, n.5, set-out, 2005. 
QUANDT et al. (1998) observaram efeitos neuromusculares, como opistótomo, movimentos de pedalagem e tremores musculares, em mais de $8 \%$ dos cães anestesiados com propofol. Esse relato vem ao encontro do observado neste estudo, no qual 8,33\% dos animais avaliados apresentaram tremores musculares na região da cabeça e nos membros, diferindo dos fatos citados por WATKINS et al. (1987), que não constataram a presença de movimentos involuntários em seu estudo, mas diferentemente deste trabalho, pré-medicou os cães com acepromazina antes da indução e manutenção anestésica com propofol.

\section{CONCLUSÕES}

Com base na metodologia proposta, foi possível concluir que as doses de infusão, utilizadas neste estudo, provocaram diminuição da temperatura retal durante o procedimento e não promoveram alterações significativas na FC e eletrocardiografia. No entanto, as reduções nas demais variáveis cardiorrespiratórias foram dependentes da dose, sendo maiores com a dose de $0,8 \mathrm{mg} / \mathrm{kg} / \mathrm{min}$, devendo esta, portanto, ser utilizada com cautela, em cães.

\section{AGRADECIMENTOS}

Os autores agradecem à Fundação de Amparo à Pesquisa do Estado de São Paulo (FAPESP), pelo apoio financeiro concedido e ao Programa de Pós-graduação em Cirurgia Veterinária da Faculdade de Ciências Agrárias e Veterinárias - FCAV.

\section{FONTE DE AQUISIÇÃO}

aDIPRIVAN - ZENECA Farmacêutica do Brasil Ltda. - São Paulo, SP, Brasil.

'Infusion Pump 670T - SAMTRONIC - São Paulo, SP, Brasil. 'DIXTAL - mod. DX - 2010LCD - Manaus, AM, Brasil (Processo FAPESP 02/04625-0).

\section{REFERÊNCIAS}

AGUIAR, A.J.A. et al. Anestesia por infusão contínua com propofol em cães após medicação pré-anestésica com levomepromazina. J Vet Anaesth, Newmarket, v.20, p.2628, 1993

ANTUNES, F. Anestesia por infusão contínua e por doses complementares de propofol em gatos pré-tratados com acepromazina. 1999. 84f. Dissertação (Mestrado em Medicina Veterinária) - Universidade Federal de Viçosa.

BUFALARI, A. et al. Comparative responses to propofol anesthesia alone with alpha 2-adrenergic medications in a canine model. Acta Vet Scand, Frederiksberg, v.37, n.2, p.187-201, 1996.

COATES, D.P. et al. Hemodynamic effects of infusion of the emulsion formulation of propofol during nitrous oxide anesthesia in humans. Anesth Analg, Baltimore, v.66, n.1, p.64-70, 1997.

DUKE,T. A new intravenous anesthetic agent: Propofol. Can Vet J, Ottawa, v.36, p.181-183, 1995.

FANTONI, D.T. Anestesia no cardiopata. In: FANTONI, D.T.; CORTOPASSI, S.R.G. Anestesia em cães e gatos. São Paulo : Roca, 2002. Cap.30, p.294-320a.

FANTONI, D.T. Recuperação pós-anestésica. In: FANTONI, D.T.; CORTOPASSI, S.R.G. Anestesia em cães e gatos. São Paulo : Roca, 2002. Cap.35, p.369-378b.

FANTONI, D.T. Anestésicos intravenosos e outros parenterais. In: SPINOSA, H.S. et al. Farmacologia aplicada à medicina veterinária. Rio de Janeiro : Guanabara Koogan 1996. Cap.11, p.114-124.

GOODCHILD, C. S.; SERRAO, J. M. Cardiovascular effects of propofol in the anaesthetizes dog. Br J Anaesth, Oxford, v. 63, n. 1, p. 87-92, 1989.

GUERRERO, P.N. Influência do monitoramento do Indíce Biespectral sobre o comportamento de variáveis cardiorrespiratórias e consumo de sevoflurano, em cães. 2003. 65f. Dissertação (Mestrado em Cirurgia Veterinária) - Faculdade de Ciências Agrárias e Veterinárias, Universidade Estadual Paulista.

HALL, L.W.; CHAMBERS, J.P. A clinical trial of propofol infusion anaesthesia in dogs. J Small Anim Pract, London, v.28, p.623-637, 1987.

KARSKI, J.M. et al. Propofol for continuos intravenous sedation after aortocoronary bypass graft surgery: dose finding study [abstract]. Can J Anaesth, Ontario, n.41, suppl.5, p.A 17, 1994.

KEEGAN, R.D.; GREENE, S.A. Cardiovascular effects of continuous two-hour propofol infusion in dogs. Comparision with isoflurane anesthesia. Vet Surg, v.22, n.6, p.537-543, 1993.

MORGAN, D.W.T.; LEGGE K. Clinical evaluation of propofol as na intravenous anesthetic agent in cats and dogs. Vet Rec, London, n.1, p.31-33, 1989.

MUIR III, W.W.; GADAWASKI, J.E. Respiratory depression and apnea induced by propofol in dogs. Am J Vet Res, Schaumburg, v.59, n.2, p.157-161, 1998.

PIRES, J.S. et al. Anestesia por infusão contínua de propofol em cães pré-medicados com acepromazina e fentanil. Cienc Rural, Santa Maria, v.30, n.5, p.829-834, 2000.

QUANDT, J.E. et al. Cardorespiratory and anesthetic of propofol and thiopental in dogs. Am J Vet Res, Schaumburg, v.59, n.9, p.1137-1143, 1998.

ROBERTS, F.L. et al. Induction and maintenance of propofol anaesthesia. Anaesthesia, London, v.43, n.1, p.14-17, 1988.

SHORT, C.E.; BUFALARI, A. Propofol anesthesia. Vet Clin North Am Small: Anim Pract, Tennessee, v.29, n.3, p.747-778, 1999 .

Ciência Rural, v.35 n.5, set-out, 2005. 
THURMON, J.C. et al. Injectable anesthetics. In: Lumb \& Jones' veterinary anesthesia. 3.ed. Philadelphia: Lippincott Williams \& Wilkins, 1996. Cap.9, p.232-233.

TILLEY, L.P. Essentials of canine and feline electrocardiographic: interpretation and treatment. 3.ed. Philadelphia : Lea \& Feabinger, 1992. 470p.
WATKINS, S.B. et al. Propofol as na intravenous anaesthetic agent in dogs. Vet Rec, Lodon, v.120, p.326-329, 1987.

WHITWAM, J.G. et al. The effects of propofol on heart rate arterial pressure and $\mathrm{A}$ and Csomatosympathetic reflexes in anesthetized dogs. Eur J Anaesthesiol, Oxford, v.17, n.1, p.57-63, 2000 